\title{
Cellulose synthase complexes: composition and regulation
}

\section{Lei Lei, Shundai Li and Ying Gu*}

The Center for LignoCellulose Structure and Formation, Department of Biochemistry and Molecular Biology, Pennsylvania State University, University Park, PA, USA

\section{Edited by:}

Seth DeBolt, University of Kentucky,

USA

\section{Reviewed by:}

Herman Höfte, Institut National de la Recherche Agronomique, France

Raymond Wightman, University of Cambridge, UK

\section{${ }^{*}$ Correspondence:}

Ying Gu, Department of Biochemistry and Molecular Biology, Pennsylvania

State University, 262 North Frear,

University Park, PA 16802, USA.

e-mail: yug13@psu.edu
Live cell imaging has greatly advanced our knowledge on the molecular mechanism by which cellulose is deposited. Both the actin and microtubule cytoskeleton are involved in assuring the proper distribution, organization, and dynamics of cellulose synthase complexes (CSCs). This review is an update on the most recent progress on the characterization of the composition, regulation, and trafficking of CSCs. With the newly identified cellulose synthase interactive protein 1 (CSI1) on hand, we begin to unveil the mystery of an intimate relationship between cellulose microfibrils and microtubules.

Keywords: terminal complexes, cellulose synthase complexes, cellulose microfibrils, microtubules, alignment hypothesis, actin

\section{INTRODUCTION}

Cellulose, in its simplest form, is composed of $\beta$-1,4-linked glucose. Cellulose synthesis captures a lot of interest not only because cellulose is a major component of plant cell walls, but also due to its potential to become one of the major resources for renewable biofuels. Many excellent reviews have highlighted contribution toward the understanding of cellulose biosynthesis (Delmer, 1999; Williamson et al., 2002; Saxena and Brown, 2005; Somerville, 2006; Crowell et al., 2010). This review will focus on the composition and regulation of cellulose synthase complexes (CSCs).

\section{VISUALIZATION OF CELLULOSE SYNTHASE COMPLEXES}

Cellulose synthase complexes, or "granules" as they were referred to at the time, were first observed by electron microscopy in 1972, signifying the origin of the visualization of the cellulose biosynthesis machinery (Robinson et al., 1972). A few years later, Brown et al. observed similar granules in both prokaryotes Acetobacter xylinum and eukaryotes Oocystis and appropriately named them "terminal complexes" (Brown and Montezinos, 1976; Brown et al., 1976). The name terminal complexes (TCs) reflects the association of complexes at the end of cellulose microfibrils as seen by electron microscopy in freeze fracture replicas. Since then, TCs have been observed in many organisms. It was speculated that TCs were the cellulose-synthesizing units long before the identification of the first cellulose synthase (CESA) gene in A. xylinus (Saxena et al., 1990) and the immuno-gold labeling of TCs using CESA antibodies (Kimura et al., 1999).

Fluorescent protein tagging has no doubt revolutionized how we visualize protein complex dynamics at the intracellular level. So far, four CESA proteins in Arabidopsis thaliana have been visualized using fluorescent protein tagging via confocal microscopy. In epidermal cells from dark grown hypocotyls, both YFP-CESA6 and GFP-CESA3 localize at the plasma membrane as distinct particles and move bidirectionally at an average speed of $270-350 \mathrm{~nm} / \mathrm{min}$ (Paredez et al., 2006; Desprez et al., 2007). These rates of movement are roughly calculated to correspond to the addition of 300-1000 glucose residues per glucan chain per minute (Paredez et al., 2006). GFP-CESA5 particles move at a similar speed of $270 \mathrm{~nm} / \mathrm{min}$ when expressed in wild type plants (Bischoff et al., 2011). Using fluorescence loss in photobleaching (FLIP), the velocities of YFP-CESA7 particles in the developing xylem of Arabidopsis were determined to be greater than $7 \mu \mathrm{m} / \mathrm{s}$, more than 1000 -fold faster than that of primary CESAs (Wightman et al., 2009). Although secondary wall biosynthesis occurs at a much faster rate than that of primary wall, it cannot account for the drastic difference between the velocities of CESAs in two types of walls. Nevertheless, with many choices of fluorescent proteins in hand and as resolution improves to molecular scales, simultaneous imaging of different CESA isoforms might reveal the composition and positional information of CESA proteins in the rosettes.

\section{CSC MODELS}

Primary cell walls are deposited during cell division and cell expansion. Secondary cell walls are deposited between primary walls and plasma membrane after the cessation of cell growth. Cellulose in the primary and secondary cell wall differs in the degree of polymerization and crystallinity. These differences may be due to the different composition and structure of primary and secondary CSCs. Because cellulose microfibrils in vascular plants are estimated to vary from 18 to 36 glucan chains and three isoforms of CESA (CESA4, 7, and 8) are required in the secondary wall formation, a heteromeric model of 18-36 CESA proteins is formulated to support the non-redundant roles of three CESA isoforms in cellulose synthesis of secondary cell walls (Scheible et al., 2001; Doblin et al., 2002; Taylor et al., 2003). For cellulose synthesis of primary cell wall, CSC is composed of CESA1, 3, 6, and 6-like proteins (CESA2, 5, and 9). It is worth noting that these CSC models are based on several assumptions and clarification on the exact composition and stoichiometry of CESAs in both primary and secondary CSCs is needed.

The distinction between primary and secondary CESAs might not be as strict as initially defined. For example, the primary CESA 
clades (CESA1, 3, 6, and 6-like) support secondary wall synthesis in Arabidopsis trichomes (Betancur et al., 2010). Another example came from the most recent findings that primary CESAs function in the formation of secondary cell walls in the seed coat (Stork et al., 2010; Mendu et al., 2011). Specifically, CESA5 is responsible for mucilage attachment and CESA2, 5, and 9 contribute toward secondary cell wall biosynthesis in the columella cells (Sullivan et al., 2011). These findings may provide insight into how CSCs evolved to fulfill their specific roles in distinct cell types.

\section{CSCs AND MICROTUBULE CYTOSKELETON}

The association between cortical microtubules and cellulose microfibril deposition has been well documented in the literature (Hepler and Newcomb, 1964; Mueller and Brown, 1980; Quader et al., 1987; Baskin, 2001; Gardiner et al., 2003). The microtubule-microfibril alignment hypothesis proposes that cortical microtubules, which lie beneath the plasma membrane of elongating cells, provide tracks for CSCs that convert glucose molecules into crystalline cellulose microfibrils (Green, 1962). The alignment hypothesis has been assessed extensively in many organisms including three widely studied green algae Closterium acerosum, Oocystis solitaria, Nitella axillaris, and land plants, e.g., Coleus blumei, oat, Arabidopsis, maize, cotton (Seagull, 1991; Baskin, 2001). Pharmacological experiments using microtubuledisrupting agents in diffusely growing cells generally support the alignment hypothesis, but discrepancies have also been noted (Seagull, 1991; Baskin, 2001; Wasteneys, 2004).

The alignment hypothesis was recently tested by visualization of microtubules and CSCs simultaneously in living plant cells. The co-alignment of CSCs and microtubules was observed in primary cell walls using a transgenic line co-expressing CFP-TUA1 and YFP-CESA6 (Paredez et al., 2006). YFP-CESA6 localizes at the plasma membrane as distinct particles, tracing linear trajectories that are coincident with the underlying microtubules. Changes in microtubule orientation by blue light excitation resulted in correlated shift in trajectories of YFP-CESA6. Complete removal of microtubules by the microtubule depolymerization agent, oryzalin, resulted in a uniform distribution of CSCs (Crowell et al., 2009; Li et al., 2012). GFP-CESA7 formed thick bands in the developing xylem of Arabidopsis, coinciding with bands of microtubules that mark the sites of secondary cell wall deposition (Gardiner et al., 2003). Localization of CSC to sites of secondary wall synthesis is also dependent upon underlying microtubules (Wightman and Turner, 2008). Within 45 min of oryzalin treatment, transverse bands labeled by YFP-CESA7 had disappeared concurrently with the loss of CFP-labeled microtubule bundles.

Two alternative models have been proposed to explain the microfibril-microtubule alignment. The direct hypothesis postulates some types of direct linkage between CESA complexes and microtubules (Heath, 1974). The indirect hypothesis, also known as the bumper model, proposes that the interaction of microtubules with plasma membrane changes membrane fluidity thereby restricting the movement of the CSCs (CESA complexes) and there is no need for direct interaction between CESA complexes and microtubules (Herth, 1980). The indirect hypothesis gained popularity and is now commonly found in textbooks
(Alberts et al., 1994) although no direct evidence supports the bumper model.

\section{CSI1: REVISIT THE ALIGNMENT HYPOTHESIS}

A yeast two-hybrid screen using the entire central cytosolic domain of CESA was used to identify proteins that interact with CESA and potentially link CSCs to the cytoskeleton. CESA interactive protein 1 (CSI1) is among several dozen putative CESA interactive proteins identified. csil null mutants displayed cell expansion defects in hypocotyls and roots, correlating with a reduction in crystalline cellulose content (Gu and Somerville, 2010; Gu et al., 2010). Red fluorescent protein tagged CSI1 (CSI1-RFP) localizes at the plasma membrane as distinct particles and travels bidirectionally at a similar velocity to GFP-CESA3. Moreover, the linear trajectories of CSI1-RFP coincide with the underlying microtubules labeled by YFP-TUA5. In the absence of CSI1, both the distribution and the motility of CESA complexes are defective and the alignment of CSCs and microtubules is disrupted (Gu et al., 2010; Bringmann et al., 2012; Li et al., 2012). These observations led to the hypothesis that CSI1 directly mediates the interaction between CSCs and microtubules (Figure 1).

An in vitro microtubule-binding assay supports the hypothesis that CSI1 is a linker protein between CSCs and microtubules. CSI1, like other conventional microtubule-binding proteins, cosedimented with polymerized tubulin (Li et al., 2012). CSI1 contains multiple tandem copies of Armadillo (ARM) repeats, degenerate protein sequence motifs for protein-protein interaction. Although CSI1 does not share sequence homology with known structural microtubule-associated proteins (MAPs), ARM repeat containing proteins have been shown to be involved in cytoskeletal functions. As a bona fide MAP, CSI1 does associate with microtubules in vivo. CSI1 is present in all land plants. It remains to be determined whether CSI1 represents a conserved mechanism for microfibril-microtubule alignment.

Though csil mutants have an expansion defect, these plants are still viable. Arabidopsis encodes two CSI1-like proteins, namely CSI2 and CSI3. The triple mutant, csilcsi2csi3 is viable but has an expansion defect that is greater than that of csil (Bringmann et al., 2012). The viability of the triple mutant might suggest that other proteins may be involved in microfibril-microtubule alignment. Additional work will be required to test this and other possibilities.

\section{CSCs AND ACTIN CYTOSKELETON}

In epidermal cells of dark grown Arabidopsis hypocotyls, actin is required for the motility and the global distribution of Golgi localized CSC populations (Crowell et al., 2009; Gutierrez et al., 2009). In this cell type, microtubules mark the site for CSC delivery to the plasma membrane for primary cell wall synthesis (Crowell et al., 2009; Gutierrez et al., 2009). Contrastingly, in xylem vessels, which are models for secondary wall synthesis, microtubules are apparently not essential in marking the sites for CSC delivery based on observations that oryzalin treatment does not prevent CSCcontaining compartments from pausing at sites of wall synthesis (Wightman and Turner, 2008). In xylem vessels transverse actin bundles are positioned close to the sites of wall synthesis and a disruption of actin by Latrunculin B results in a loss of transverse 

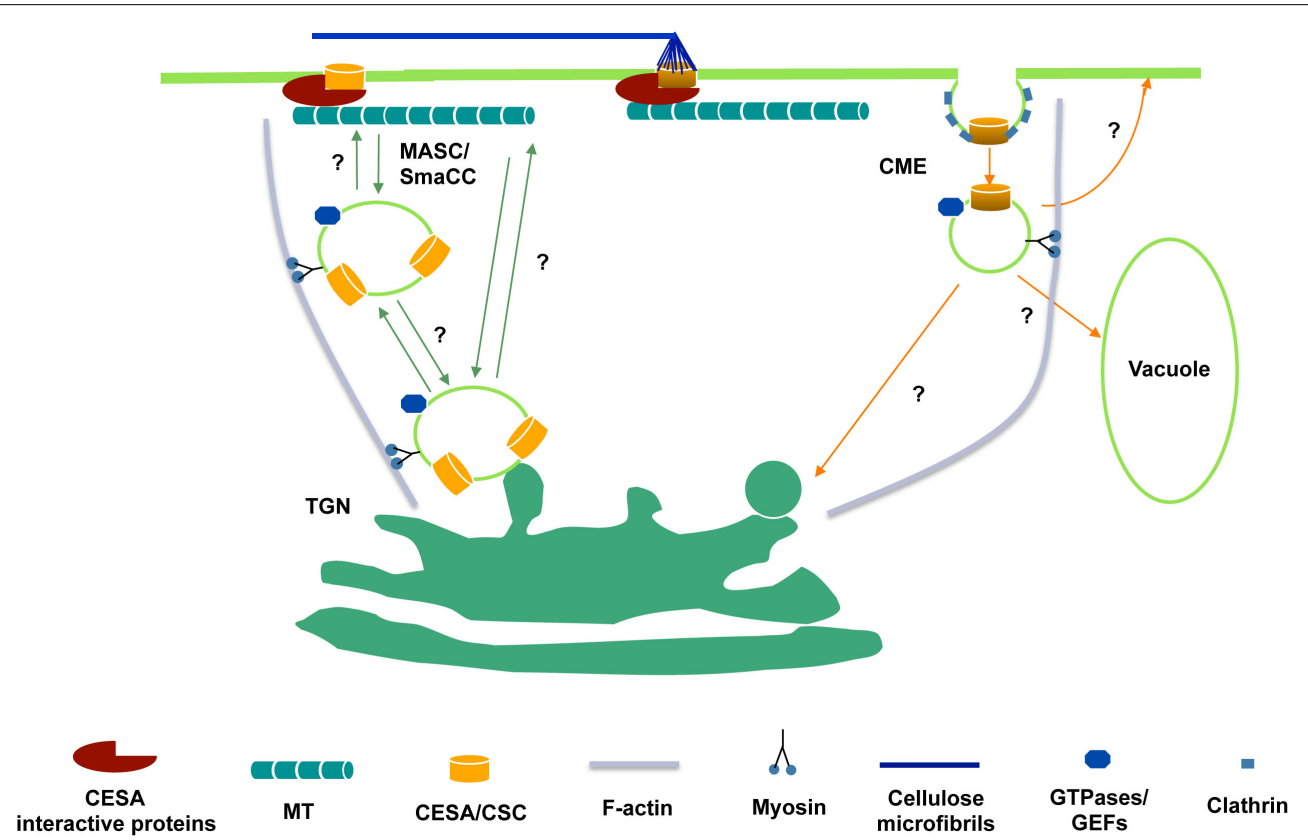

FIGURE 1 | Hypothetical schematic diagram of the trafficking of CSCs to and from the plasma membrane. CSCs are presumably synthesized in ER and delivered to Golgi for assembly. From Golgi to plasma membrane, delivery may occur directly from the trans-Golgi network (TGN) or through an intermediate compartment such as the MASC/SmaCC. MASCs/SmaCCs may also act as an internalization or recycling compartment. Also, CESA proteins may be internalized by clathrin-mediated endocytosis (CME). The association of CESA interactive proteins with CSCs may occur before the insertion event. At the plasma membrane, CESA interactive proteins, e.g., CSI1, bridge between CSCs and microtubules and enforce the co-alignment of newly synthesized cellulose microfibrils and cortical microtubules.
CSC bands. These observations led to a hypothesis that transverse actin bands, rather than microtubules, mark CSC delivery sites at the cell membrane in xylem vessels (Wightman and Turner, 2008). If this hypothesis holds true, then the mechanism for CSC delivery might be different for primary walls vs secondary walls.

During secondary wall synthesis in cotton fibers, actin microfilaments reorient from an axial pattern to a helical pattern, correlating with the secondary wall deposition. Disruption of the actin cytoskeleton by cytochalasins in cotton fibers and Zinnia treachery elements resulted in the disorganization of cellulose microfibrils (Seagull, 1990). Microtubules were observed to reorient upon disruption of the actin microfilament array, and it was therefore proposed that the actin cytoskeleton may contribute to cell elongation through the interaction between actin microfilaments and microtubules (Wasteneys and Collings, 2004). The cross-talk between actin and microtubules has been demonstrated mostly by pharmacological studies where stabilizing or disrupting one element affects the other (Collings, 2008). Recently, the dynamic association between actin microfilaments and microtubules was confirmed in interphase plant cells using an F-actin and microtubule dual-labeled line (Sampathkumar et al., 2011). In animal cells, molecular linker proteins such as MAP1 and 2, coronin, ERM proteins, and dynein support the interaction between actin and microtubules. However, plants lack counterparts of any of the mammalian linker proteins. The evidence for functional interactions with both microtubules and microfilaments is still missing for potential candidates such as PLD, LIM, and dynamin-related proteins (Collings, 2008).

\section{CSC TRAFFICKING AND RECYCLING}

YFP-CESA6 and GFP-CESA3 label at least three distinct populations. In addition to discrete particles at the focal plane of plasma membrane and donut-shaped Golgi compartments, CESAs also label a small compartment termed SmaCCs (small CESA compartments) or MASCs (microtubule-associated cellulose synthase compartments). The plasma membrane localized particles presumably represent functional complexes that convert glucose molecules into cellulose microfibrils. Other CESA associated compartments have a role in CESA delivery, internalization, and/or recycling event. SmaCCs/MASCs accumulate in the cell cortex upon osmotic stress, protein synthesis inhibition, or cellulose synthesis inhibition (Crowell et al., 2009; Gutierrez et al., 2009). In untreated cells, SmaCCs/MASCs are present in fully elongated cells $10 \mathrm{~mm}$ below the apical hook where CSCs are nearly absent (Crowell et al., 2009). Perturbation of CESA activity by either osmotic stress or cellulose synthesis inhibition resulted in prolongation of SmaCCs/MASCs-microtubule interactions, which appeared to be important for directing the plasma membrane delivery of CSCs. However, it remains to be determined whether SmaCCs/MASCs represents an internalization/recycling compartments or delivery compartments or both (Bashline et al., 2011). Disruption of clathrin-mediated endocytosis (CME) may have an effect on the composition of cell walls ( $\mathrm{Gu}$, unpublished data). A dynamin-like protein, DRP1A was reported to have a role in CME (Konopka and Bednarek, 2008). The defect in endocytosis in rsw9, which contains a mutation in DRP1A, resulted in the cellulose deficiency and cell elongation defect (Collings et al., 2008). CME might play 
a role in internalization and recycling of CSC components that are no longer functional (Figure 1).

\section{REGULATION OF CELLULOSE SYNTHESIS}

The rate of cellulose synthesis is affected by many factors including hormones, light, mechanical stimuli, nutrition, and interactions with the cytoskeleton. These factors may influence the cellulose deposition by affecting the levels of substrate, the abundance of CSCs in the PM, and/or the activity of CSCs. UDP-glucose, produced presumably by sucrose synthase (SUSY), is the substrate of cellulose synthesis. The most popular model, presented by Haigler et al. stated that UDP-glucose is channeled to CSCs in the plasma membrane through SUSY. Consistent with this idea, suppression of SUSY in carrot and potato tubers by expressing an antisense version of SUSY led to a reduction in cellulose formation (Tang and Sturm, 1999; Haigler et al., 2001). However, quadruple mutant plants that lack four of six isoforms of SUSY (sus1/sus $2 /$ sus $3 /$ sus 4 ) are normal in cell wall structure and cellulose content. These mutants lack SUS activity in all cell types except the phloem. The double mutant of the two phloem specific SUSYs (sus5/sus6) were also normal except for reduced amounts of callose in the sieve plates (Barratt et al., 2009). In contrast to the findings of Barratt et al. recent analysis using optimal $\mathrm{pH}$ conditions revealed SUS activity in leaves and stems of sus $1 /$ sus $2 /$ sus $3 /$ sus 4 and sus $5 /$ sus 6 plants is $85 \%$ of that of wild type plants (Baroja-Fernandez et al., 2012). Further analysis of sextuple mutants will be necessary to clarify whether SUS provides the primary route to supply carbon for cellulose and starch biosynthesis in Arabidopsis.

Bacterium cellulose synthesis requires the activation of bcsA, the catalytic subunit of cellulose synthase, through c-di-GMP (Ross et al., 1987). It appears to be a specific type of regulation in bacteria since plants does not have c-di-GMP. It has been proposed that phosphorylation might have a potential role in regulating CESA activity in plants (Somerville, 2006). Phosphoproteomics of Arabidopsis suspension culture cells revealed that CESA 1, CESA3, and CESA5 were phosphorylated at a number of sites clustered in the N-terminal variable region (Nuhse et al., 2004). The phosphorylation of secondary CESAs (CESA4 and CESA7) was also reported at a similar region (Taylor, 2007). Recently, the role of phosphorylation was tested for CESA1 in Arabidopsis (Chen et al., 2010). To modify the phosphorylation status of CESA1, six putative phosphorylation sites containing serine $(S)$ or threonine (T) were substituted with alanine (A) to prevent phosphorylation or glutamine (E) to mimic phosphorylation and the resulting constructs were transformed into $r s w 1$ mutant background. Abolishing the putative phosphorylation sites for T165, T166, and S167 did not rescue the defect on growth anisotropy (i.e., radial swelling of cells). The other three pairs of mutations (S162, S686, and S688) recovered the growth anisotropic defect in cesa $1^{r s w 1}$. The CSC dynamics were examined by introducing YFP-CESA6 into the

\section{REFERENCES}

Alberts, B., Bray, D., Lewis, J., Raff, M., Roberts, K., and Watson, J. D. (1994). "Cell juctions, cell adhesion, and the extracellular matrix," in Molecular Biology of the Cell, ed. S. Gibbs (New York: Garland Science), 1124.
Baroja-Fernandez, E., Munoz, F. J., Li, J., Bahaji, A., Almagro, G., Montero, M., Etxeberria, E., Hidalgo, M., Sesma, M. T., and Pozueta-Romero, J. (2012). Sucrose synthase activity in the sus1/sus2/sus3/sus4 Arabidopsis mutant is sufficient to support

above transgenic lines. Transgenic lines with reduced cell elongation and loss of cell anisotropy also showed asymmetric motility of CSCs in contrast to bidirectional movement in wild type cells. More interestingly, the asymmetric motility of CSCs in T165E and T165A was recovered by removal of microtubules using depolymerizing agent oryzalin. These observations led to the hypothesis that phosphorylation of CESA differentially affects a polar interaction with microtubules, resulting in velocity differences of CSCs on two sides of a microtubule.

The role of phosphorylation on motility of CSCs was also tested using CESA5. CESA5, presumably redundant with CESA6, did not fully complement the growth defect in cesa $6^{p r c 1-1}$ when it was expressed under the native promoter of CESA6. When putative phosphorylation sites at Ser122, Ser126, Ser229, and Ser230 were all concomitantly substituted with glutamine, the resulting construct fully complemented the growth defect and reduced motility of CSCs in cesa $6^{p^{r c l-1}}$ (Bischoff et al., 2011). Based on these observations, authors propose that phosphorylation is required to render CESA5 functionally equivalent to CESA6. Another CESA6like protein, CESA2 was previously shown to fully complement the growth defect in cesa $6^{\text {prcl-1 }}$ using a similar promoter swap strategy (Persson et al., 2007). Apparently, CESA2 may not require phosphorylation to substitute function of CESA6 because CESA2 does not have predicted phosphorylation sites at similar positions as CESA5.

\section{CONCLUSION}

Understanding the complex process of cellulose synthesis will be important for optimizing the use of cellulose as a renewable energy source. Although significant progress has been made in understanding the structure and regulation of CSCs, many fundamental aspects remain to be addressed. For example, the exact composition of the CSC is not known, nor do we know about the interaction pattern among isoforms of CESAs. The crystal structure of plant CESAs remains unsolved. In vitro synthesis of cellulose with plant CESAs is still at its infancy stage due to various technical difficulties. The exact molecular details of how microtubules guide the deposition of cellulose microfibrils are still missing. Cellulose biosynthesis is an exciting area of study with lots of challenges and opportunities. Advances in biochemical characterization and cell imaging tools in conjunction with the power of plant genetics will one day allow us to reconstitute an intact and fully functional cellulose synthesis machinery.

\section{ACKNOWLEDGMENTS}

Lei Lei, Shundai Li, and Ying Gu are in part supported by the Center for LignoCellulose Structure and Formation, an Energy Frontier Research Center funded by the U.S. Department of Energy, Office of Science under Award Number DE-SC0001090. Ying Gu is supported by grants from National Science Foundation (1121375).

normal cellulose and starch production. Proc. Natl. Acad. Sci. U.S.A. 109, 321-326.

Barratt, D. H., Derbyshire, P., Findlay, K., Pike, M., Wellner, N., Lunn, J., Feil, R., Simpson, C., Maule, A. J., and Smith, A. M. (2009). Normal growth of Arabidopsis requires cytosolic invertase but not sucrose synthase. Proc. Natl. Acad. Sci. U.S.A. 106, 13124-13129.

Bashline, L., Du, J., and Gu, Y. (2011). The trafficking and behavior of cellulose synthase and a 
glimpse of potential cellulose synthesis regulators. Front. Biol. 6:5. doi:10.1007/s11515-011-1161-3

Baskin, T. I. (2001). On the alignment of cellulose microfibrils by cortical microtubules: a review and a model. Protoplasma 215, 150-171.

Betancur, L., Singh, B., Rapp, R. A., Wendel, J. F., Marks, M. D., Roberts, A. W., and Haigler, C. H. (2010). Phylogenetically distinct cellulose synthase genes support secondary wall thickening in Arabidopsis shoot trichomes and cotton fiber. J. Integr. Plant Biol. 52, 205-220.

Bischoff, V., Desprez, T., Mouille, G., Vernhettes, S., Gonneau, M., and Hofte, H. (2011). Phytochrome regulation of cellulose synthesis in Arabidopsis. Curr. Biol. 21, 1822-1827.

Bringmann, M., Li, E., Sampathkumar, A., Kocabek, T., Hauser, M. T., and Persson, S. (2012). POMPOM2/cellulose synthase interacting1 is essential for the functional association of cellulose synthase and microtubules in Arabidopsis. Plant Cell 24, 163-177.

Brown, R. M. Jr., and Montezinos, D. (1976). Cellulose microfibrils: visualization of biosynthetic and orienting complexes in association with the plasma membrane. Proc. Natl. Acad. Sci. U.S.A. 73, 143-147.

Brown, R. M. Jr., Willison, J. H., and Richardson, C. L. (1976). Cellulose biosynthesis in Acetobacter xylinum: visualization of the site of synthesis and direct measurement of the in vivo process. Proc. Natl. Acad. Sci. U.S.A. 73, 4565-4569.

Chen, S., Ehrhardt, D. W., and Somerville, C. R. (2010). Mutations of cellulose synthase (CESA1) phosphorylation sites modulate anisotropic cell expansion and bidirectional mobility of cellulose synthase. Proc. Natl. Acad. Sci. U.S.A. 107, 17188-17193.

Collings, D. A. (2008). "Crossed wires: interactions and cross-talk between the microtubule and microfilament networks in plants," in Plant Cell Monographs: Plant Microtubules, Development, and Flexibility, ed. P. Nick (Berlin: Springer), 47-82.

Collings, D. A., Gebbie, L. K., Howles, P. A., Hurley, U. A., Birch, R. J., Cork, A. H., Hocart, C. H., Arioli, T., and Williamson, R. E. (2008). Arabidopsis dynamin-like protein DRP1A: a null mutant with widespread defects in endocytosis, cellulose synthesis, cytokinesis, and cell expansion. J. Exp. Bot. 59, 361-376.

Crowell, E. F., Bischoff, V., Desprez, T., Rolland, A., Stierhof, Y. D., Schumacher, K., Gonneau, M., Hofte, H., and Vernhettes, S. (2009). Pausing of Golgi bodies on microtubules regulates secretion of cellulose synthase complexes in Arabidopsis. Plant Cell 21, 1141-1154.

Crowell, E. F., Gonneau, M., Stierhof, Y. D., Hofte, H., and Vernhettes, S. (2010). Regulated trafficking of cellulose synthases. Curr. Opin. Plant Biol. 13, 700-705.

Delmer, D. P. (1999). Cellulose biosynthesis: exciting times for a difficult field of study. Annu. Rev. Plant Physiol. Plant Mol. Biol. 50, 245-276.

Desprez, T., Juraniec, M., Crowell, E. F., Jouy, H., Pochylova, Z., Parcy, F., Hofte, H., Gonneau, M., and Vernhettes, S. (2007). Organization of cellulose synthase complexes involved in primary cell wall synthesis in Arabidopsis thaliana. Proc. Natl. Acad. Sci. U.S.A. 104, 15572-15577.

Doblin, M. S., Kurek, I., Jacob-Wilk, D., and Delmer, D. P. (2002). Cellulose biosynthesis in plants: from genes to rosettes. Plant Cell Physiol. 43, 1407-1420.

Gardiner, J. C., Taylor, N. G., and Turner, S. R. (2003). Control of cellulose synthase complex localization in developing xylem. Plant Cell 15, 1740-1748.

Green, P. B. (1962). Mechanism for plant cellular morphogenesis. Science 138, 1404-1405.

Gu, Y., Kaplinsky, N., Bringmann, M., Cobb, A., Carroll, A., Sampathkumar, A., Baskin, T. I., Persson, S., and Somerville, C. R. (2010). Identification of a cellulose synthaseassociated protein required for cellulose biosynthesis. Proc. Natl. Acad. Sci. U.S.A. 107, 12866-12871.

Gu, Y., and Somerville, C. (2010). Cellulose synthase interacting protein: a new factor in cellulose synthesis. Plant Signal. Behav. 5, 1571-1574.

Gutierrez, R., Lindeboom, J. J., Paredez, A. R., Emons, A. M., and Ehrhardt, D. W. (2009). Arabidopsis cortical microtubules position cellulose synthase delivery to the plasma membrane and interact with cellulose synthase trafficking compartments. Nat. Cell Biol. 11, 797-806.

Haigler, C. H., Ivanova-Datcheva, M., Hogan, P. S., Salnikov, V. V., Hwang, S., Martin, K., and Delmer, D. P. (2001). Carbon partitioning to cellulose synthesis. Plant Mol. Biol. 47, 29-51.

Heath, I. B. (1974). A unified hypothesis for the role of membrane bound enzyme complexes and microtubules in plant cell wall synthesis. J. Theor. Biol. 48, 445-449.

Hepler, P. K., and Newcomb, E. H. (1964). Microtubules and fibrils in the cytoplasm of coleus cells undergoing secondary wall deposition. $J$. Cell Biol. 20, 529-532.

Herth, W. (1980). Calcofluor white and Congo red inhibit chitin microfibril assembly of Poterioochromonas: evidence for a gap between polymerization and microfibril formation. $J$. Cell Biol. 87, 442-450.

Kimura, S., Laosinchai, W., Itoh, T., Cui, X., Linder, C. R., and Brown, R. M. Jr. (1999). Immunogold labeling of rosette terminal cellulosesynthesizing complexes in the vascular plant vigna angularis. Plant Cell 11, 2075-2086.

Konopka, C. A., and Bednarek, S. Y. (2008). Comparison of the dynamics and functional redundancy of the Arabidopsis dynamin-related isoforms DRP1A and DRP1C during plant development. Plant Physiol. 147, 1590-1602.

Li, S., Lei, L., Somerville, C., and Gu, Y. (2012). Cellulose synthase interactive protein 1 (CSI1) links microtubules and cellulose synthase complexes. Proc. Natl. Acad. Sci. U.S.A. 109, 185-190.

Mendu, V., Griffiths, J. S., Persson, S. Stork, J., Downie, A. B., Voiniciuc, C., Haughn, G. W., and DeBolt, S. (2011). Subfunctionalization of cellulose synthases in seed coat epidermal cells mediates secondary radial wall synthesis and mucilage attachment. Plant Physiol. 157, 441-453.

Mueller, S. C., and Brown, R. M. Jr. (1980). Evidence for an intramembrane component associated with a cellulose microfibril-synthesizing complex in higher plants. J. Cell Biol. 84, 315-326.

Nuhse, T. S., Stensballe, A., Jensen, O. N. and Peck, S. C. (2004). Phosphoproteomics of the Arabidopsis plasma membrane and a new phosphorylation site database. Plant Cell 16, 2394-2405.

Paredez, A. R., Somerville, C. R., and Ehrhardt, D. W. (2006). Visualization of cellulose synthase demonstrates functional association with microtubules. Science 312, 1491-1495.

Persson, S., Paredez, A., Carroll, A., Palsdottir, H., Doblin, M., Poindexter, P., Khitrov, N., Auer, M., and Somerville, C. R. (2007). Genetic evidence for three unique components in primary cell-wall cellulose synthase complexes in Arabidopsis. Proc. Natl. Acad. Sci. U.S.A. 104, 15566-15571.

Quader, H., Herth, W., Ryser, U., and Schnepf, E. (1987). Cytoskeletal elements in cotton seed hair development in vitro: their possible regulatory role in cell wall organization. Protoplasma 137, 56-62.

Robinson, D. G., White, R. K., and Preston, R. D. (1972). Fine structure of swarmers of Cladophora and Chaetomorpha. III. Wall synthesis and development. Planta 107, 131-144.

Ross, P., Weinhouse, H., Aloni, Y., Michaeli, D., Weinberger-Ohana, P., Mayer, R., Braun, S., de Vroom, E., van der Marel, G. A., van Boom, J. H., and Benziman, M. (1987). Regulation of cellulose synthesis in Acetobacter xylinum by cyclic diguanylic acid. Nature 325, 279-281.

Sampathkumar, A., Lindeboom, J. J., Debolt, S., Gutierrez, R., Ehrhardt, D. W., Ketelaar, T., and Persson, S. (2011). Live cell imaging reveals structural associations between the actin and microtubule cytoskeleton in Arabidopsis. Plant Cell 23, 2302-2313.

Saxena, I. M., and Brown, R. M. Jr. (2005). Cellulose biosynthesis: current views and evolving concepts. Ann. Bot. 96, 9-21.

Saxena, I. M., Lin, F. C., and Brown, R. M. Jr. (1990). Cloning and sequencing of the cellulose synthase catalytic subunit gene of Acetobacter xylinum. Plant Mol. Biol. 15, 673-683.

Scheible, W. R., Eshed, R., Richmond, T., Delmer, D., and Somerville, C. (2001). Modifications of cellulose synthase confer resistance to isoxaben and thiazolidinone herbicides in Arabidopsis Ixr1 mutants. Proc. Natl. Acad. Sci. U.S.A. 98, 10079-10084.

Seagull, R. W. (1990). The effects of microtubule and microfilament disrupting agents on cytoskeletal arrays and wall deposition in developing cotton fibers. Protoplasma 159, 44-59.

Seagull, R. W. (1991). "Role of the cytoskeletal elements in organized wall microfibril deposition," in Biosynthesis and Biodegradation of Cellulose, eds C. H. Haigler and P. J. Weimer (New York: Marcel Dekker), 143-163.

Somerville, C. (2006). Cellulose synthesis in higher plants. Annu. Rev. Cell Dev. Biol. 22, 53-78.

Stork, J., Harris, D., Griffiths, J., Williams, B., Beisson, F., Li-Beisson, Y., Mendu, V., Haughn, G., and DeBolt, S. (2010). Cellulose synthase 9 serves a nonredundant role in secondary cell wall synthesis in Arabidopsis epidermal testa cells. Plant Physiol. 153, 580-589.

Sullivan, S., Ralet, M. C., Berger, A., Diatloff, E., Bischoff, V., Gonneau, M., Marion-Poll, A., and North, H. 
M. (2011). CESA5 is required for the synthesis of cellulose with a role in structuring the adherent mucilage of Arabidopsis seeds. Plant Physiol. 156, 1725-1739.

Tang, G. Q., and Sturm, A. (1999). Antisense repression of sucrose synthase in carrot (Daucus carota L.) affects growth rather than sucrose partitioning. Plant Mol. Biol. 41, 465-479.

Taylor, N. G. (2007). Identification of cellulose synthase AtCesA7 (IRX3) in vivo phosphorylation sites - a potential role in regulating protein degradation. Plant Mol. Biol. 64, 161-171.

Taylor, N. G., Howells, R. M., Huttly, A. K., Vickers, K., and Turner, S.
R. (2003). Interactions among three distinct CesA proteins essential for cellulose synthesis. Proc. Natl. Acad. Sci. U.S.A. 100, 1450-1455.

Wasteneys, G. O. (2004). Progress in understanding the role of microtubules in plant cells. Curr. Opin. Plant Biol. 7, 651-660.

Wasteneys, G. O., and Collings, D. A. (2004). "Expanding beyond the great divide: the cytoskeleton and axial growth," in The Plant Cytoskeleton in Cell Differentiation and Development, ed. P. J. Hussey (Victoria: Blackwell), 83-115.

Wightman, R., Marshall, R., and Turner, S. R. (2009). A cellulose synthase-containing compartment moves rapidly beneath sites of secondary wall synthesis. Plant Cell Physiol. 50, 584-594.

Wightman, R., and Turner, S. R. (2008). The roles of the cytoskeleton during cellulose deposition at the secondary cell wall. Plant J. 54, 794-805.

Williamson, R. E., Burn, J. E., and Hocart, C. H. (2002). Towards the mechanism of cellulose synthesis. Trends Plant Sci. 7, 461-467.

Conflict of Interest Statement: The authors declare that the research was conducted in the absence of any commercial or financial relationships that could be construed as a potential conflict of interest.
Received: 23 January 2012; accepted: 02 April 2012; published online: 17 April 2012.

Citation: Lei L, Li S and Gu Y (2012) Cellulose synthase complexes: composition and regulation. Front. Plant Sci. 3:75. doi: 10.3389/fpls.2012.00075

This article was submitted to Frontiers in Plant Physiology, a specialty of Frontiers in Plant Science.

Copyright (C) 2012 Lei, Li and Gu. This is an open-access article distributed under the terms of the Creative Commons Attribution Non Commercial License, which permits non-commercial use, distribution, and reproduction in other forums, provided the original authors and source are credited. 